Cân Schweizerische Zeitschrift für

Swiss Journal of Integrative Medicine

Brigitte Ausfeld-Hafter

\title{
Fortbildung
}

Schweiz Z Ganzheitsmed 2012;24:216-217

DOI: 10.1159/000341159

\section{Die Kollegiale Instanz für Komplementärmedizin KIKOM als eines der führenden Forschungszentren für Komplementärmedizin}

Bericht über die Antrittsvorlesung (Abb. 1-4) von Frau PD Dr. med. Ursula Wolf, Dozentin für Anthroposophisch Erweiterte Medizin an der KIKOM, über das Thema «Komplementärmedizin im Spiegel der Forschung» vom 18. April 2012.

tive Medizin kombiniert die konventionelle und die komplementäre Medizin.

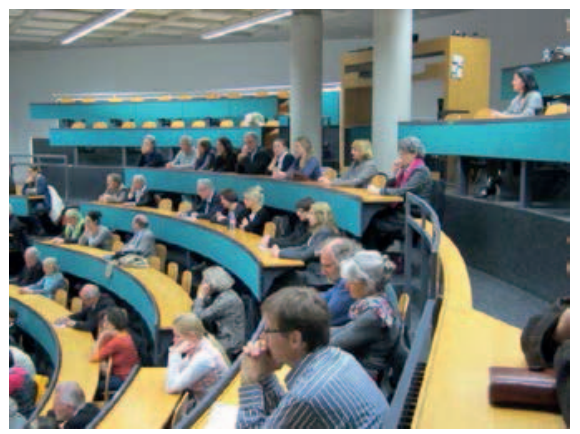

Abb. 1. Teilnehmer der Antrittsvorlesung in Bern.

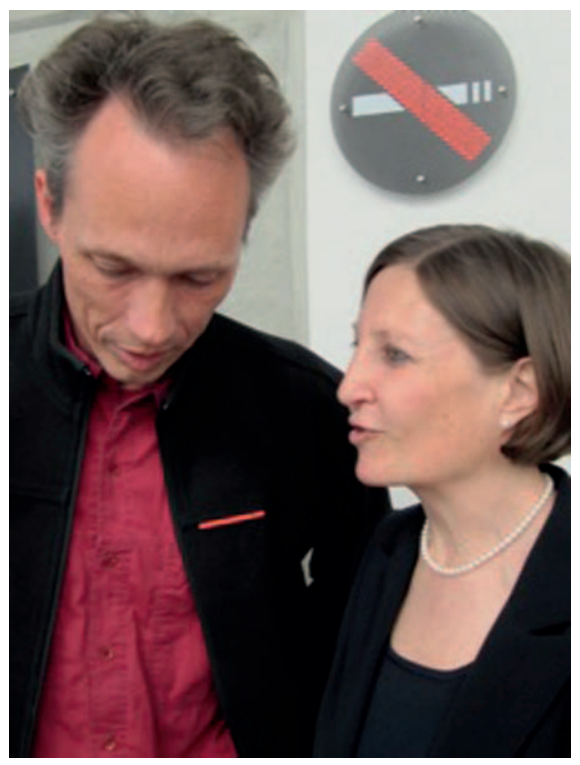

Abb. 2. Dr. sc. nat. Stephan Baumgartner und PD Dr. Ursula Wolf beim Apéro.
Generelle Aspekte der Forschung und ausgewählte Projekte

Nebst einigen Worten zur EvidenceBased Medicine (EBM) wurden auch die in der Komplementärmedizin möglichen Forschungsmethoden erwähnt. Und gerade hier liegt die Stärke der neuen Privatdozentin, kann sie

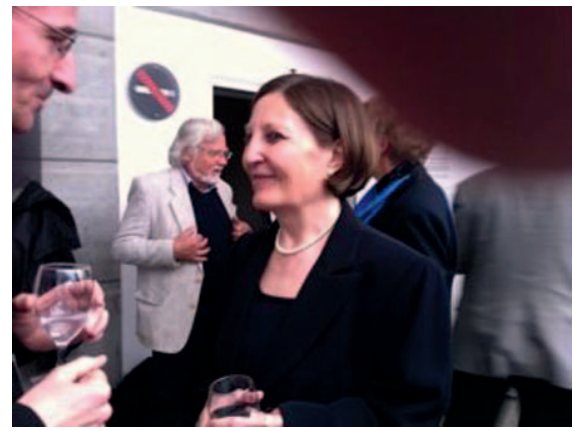

Abb. 3. PD Dr. Ursula Wolf im Gespräch mit den Teilnehmern.

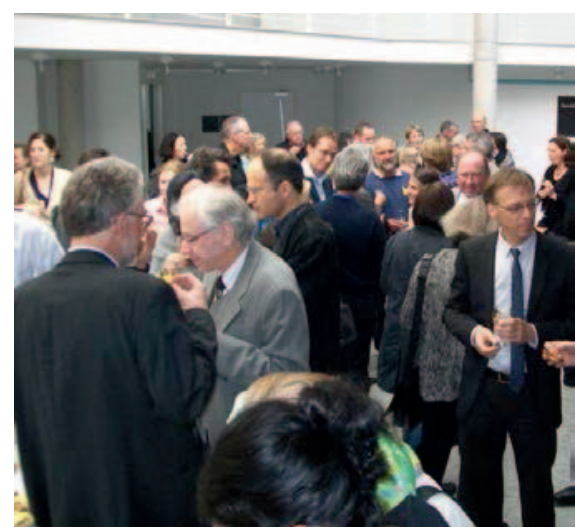

Abb. 4. Reger Andrang beim Apéro. begrifflich festgelegt, und die Integra-

Allgemeines zur

Komplementärmedizin

Zunächst ging PD Dr. Wolf auf die Definitionen der verschiedenen Begriffe ein: So wurde die Komplementärmedizin mit «sowohl als auch», also ergänzend zur konventionellen Medizin, beschrieben. Alternative Medizin ist als «entweder/oder»

Zum ersten Mal in der Schweiz hat
sich eine Ärztin in Komplementärmedizin habilitiert. Das bedeutet einen Meilenstein in der Geschichte der Medizin und ein historisches Ereignis, wie der Dozent der Klassischen Homöopathie, Dr. med. Martin FreiEr gab einen kurzen Abriss über den tätig ist und seit 2009 dem Departete Medizin vorsteht. Ihre Stärke liegt neben den von ihr ausgerichteten in-
teressanten Vorlesungen und der $\mathrm{Pa}$ tientenbetreuung in der qualitativen State-of-the-art-Methoden Wing mit Dr. Wolf es ausdrückte, ist sie «in beidem zu Hause», weil sie nebst komplementärmedizinischer Forschung auch konventionell medizinische Arbeiten verfasste.

\section{KARGER}

Fax +497614520714 Information@Karger.d www.karger.com (c) 2012 S. Karger GmbH, Freiburg www.karger.com/szg
Dr. med. Brigitte Ausfeld-Hafter

Dozentin für TCM/Akupunktur

Universität Bern, Kollegiale Instanz für Komplementärmedizin KIKOM

Imhoof-Pavillon, Inselspital, 3010 Bern, Schweiz

Tel. +41 31 632-9758, Fax -4262

brigitte.ausfeld-hafter@kikom.unibe.ch 
doch alle Studiendesigns und jede Methode in der Komplementärmedizin zur Anwendung bringen.

Vieles war über die Forschungstätigkeit von PD Dr. Wolf zu hören, die von ihr anhand einer geschickten Auswahl aus 35 in Zeitschriften mit Peer-Review-Verfahren erschienenen Originalarbeiten beschrieben wurde. Nebst den «Health Technology Assessment»-Berichten, die sie mit anderen Autoren zusammen verfasste, ging sie auf die zurzeit stattfindende klinische Prüfung von Stibium metallicum praeparatum als blutstillende Subs$\operatorname{tanz}$ ein. $\mathrm{Zu}$ diesem Thema wurden bereits 3 Vorstudien durchgeführt, die zeigen, dass ein in der Praxis bewährtes Mittel im Labor nachgeprüft und daraufhin in einem klinischen Versuch wissenschaftlich überprüft werden kann. Oder mit ihren Worten ausgedrückt: von der klinischen Praxis über die Laborbank - und zurück («from bedside to bench and back»).

Auch die therapeutische Sprachgestaltung und ihre möglichen Effekte auf wichtige physiologische Parameter hat PD Dr. Wolf untersucht.
Dass dabei auch die NahinfrarotSpektrophotometrie zur Anwendung kam, zeigt auf, dass PD Dr. Wolf sich auch mit konventionellen Forschungsmethoden beschäftigt.

Ein gut eingeführtes Pilotprojekt ist die Versorgungsforschung, nämlich das Netzwerk Onkologische Rehabilitation, das in Zusammenarbeit mit dem Spital Thun durchgeführt wird. Durch andere Projekte ist Frau PD Dr. Ursula Wolf national und international zudem bestens vernetzt.

\section{Abschluss und Ausblick}

Die Komplementärmedizin ist ein aufstrebendes Gebiet, das im Gesundheitswesen optimiert werden sollte. So ist Forschung mit hoher Qualität notwendig, die mit besserer Nachwuchsförderung und geeigneter und ausreichender Finanzierung in den kommenden Jahren vorangetrieben werden muss. Und wie im Titel angetönt, ist die KIKOM in den letzten Jahren zu einem der führendenForschungszentrenfür Komplementärmedizin avanciert.
Die Antrittsvorlesung wurde von Frau PD Dr. Ursula Wolf in würdigem Rahmen und im Beisein einer grossen Anzahl Interessierter an der Universität Bern gehalten. Dass die kulinarischen Genüsse am Ende der Veranstaltung aus ihrer früheren Heimat, nämlich aus Richterswil, stammten und von ihr bekannten Geschäftsinhabern ausgerichtet wurden, beendete den Abend wirkungsvoll. Auch die dabei möglichen Gespräche mit den an Komplementärmedizin Interessierten - es waren auch Professoren der konventionellen Medizin mit dabei - erfreute die Berichterstatterin.

Die Chinesen sagen: «Wenn der Wind des Wandels weht, dann bauen die einen Menschen Mauern, die anderen aber Windmühlen.» Den Erfolg der Habilitation, den wir mit PD Dr. Wolf an diesem Abend feiern durften, ist durch ihr stetes Bauen an Windmühlen bedingt. Wir von der KIKOM gratulieren und wünschen ihr noch viele erfolgreiche Jahre - und freuen uns auf weitere gemeinsam erbaute Windmühlen. 